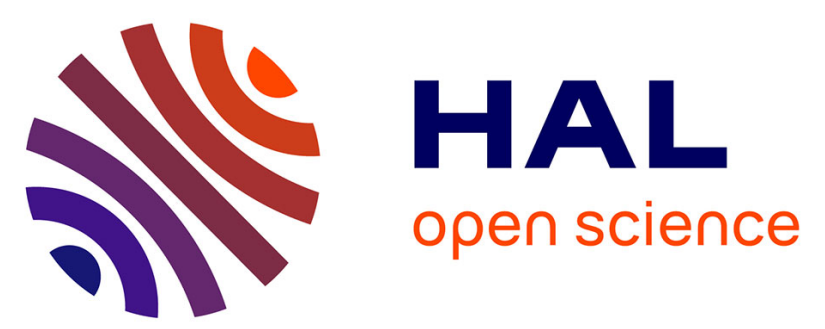

\title{
Particle image velocimetry measurements of vortex rings head-on collision with a heated vertical plate
}

Gabriel Arévalo, Rodrigo H. Hernández, Christian Nicot, Frédéric Plaza

\section{To cite this version:}

Gabriel Arévalo, Rodrigo H. Hernández, Christian Nicot, Frédéric Plaza. Particle image velocimetry measurements of vortex rings head-on collision with a heated vertical plate. Physics of Fluids, 2010, 22, pp.053604. 10.1063/1.3410800 . hal-00565998

\section{HAL Id: hal-00565998 \\ https://hal.science/hal-00565998}

Submitted on 8 Jun 2012

HAL is a multi-disciplinary open access archive for the deposit and dissemination of scientific research documents, whether they are published or not. The documents may come from teaching and research institutions in France or abroad, or from public or private research centers.
L'archive ouverte pluridisciplinaire HAL, est destinée au dépôt et à la diffusion de documents scientifiques de niveau recherche, publiés ou non, émanant des établissements d'enseignement et de recherche français ou étrangers, des laboratoires publics ou privés. 


\title{
Particle image velocimetry measurements of vortex rings head-on collision with a heated vertical plate
}

\author{
G. Arévalo, ${ }^{1}$ R. H. Hernández, ${ }^{1, a)}$ C. Nicot ${ }^{2}$ and F. Plaza ${ }^{2}$ \\ ${ }^{1}$ Departamento de Ingeniería Mecánica, LEAF-NL, Universidad de Chile, Casilla 2777, Santiago, Chile \\ ${ }^{2}$ Laboratoire de Mecanique des Fluides et d'Acoustique, CNRS Université de Lyon-Ecole Centrale \\ de Lyon-Université Lyon I-INSA Lyon E.C.L., 36 Avenue Guy de Collongue, F-69314 Ecully, France
}

(Received 8 January 2010; accepted 8 March 2010; published online 20 May 2010)

\begin{abstract}
We report particle image velocimetry measurements of the collision of a vortex ring with a heated wall kept at constant temperature. We consider the case when both the vortex ring and the thermal boundary layer generated by the vertical heated wall are stable and laminar prior to any interaction. The impingement process can be divided into two parts. (i) A ring-driven stage, where the vortex ring grows in diameter while approaching the wall and therefore it sweeps progressively an increased surface on the wall. (ii) A boundary layer-driven stage, where the vortex ring moves upward due to the thermal convective motion generated by the heated wall. In some cases, the head-on collision triggers the ring's azimuthal instability as revealed by the formation of vortical structures arranged on a wavy starlike pattern and confirmed by flow visualizations. A single collision generates important velocity gradients and shear stresses along the wall accompanied with the creation of local vorticity normal to the vertical heated wall. Peak wall shear stresses occur near the point of impact of the vortex ring core. (C) 2010 American Institute of Physics.
\end{abstract}

[doi:10.1063/1.3410800]

\section{INTRODUCTION}

The purpose of this work was to measure the vector velocity field during the collision of a vortex ring with a heated wall, to determine local vorticity and shear stresses as the basic ingredients in the mechanism which leads to an improvement of the heat transfer from the heated wall into the surrounding environment. ${ }^{1}$ The mechanism has revealed particular features that make the vortex ring a realistic candidate to enhance heat transfer from small electronic devices. A simplified situation involving this collision mechanism has been reported by other authors providing important insight on vortex stretching ${ }^{2-4}$ and pressure gradients ${ }^{4,5}$ at the wall, where the vortex core could be responsible for local and peaked shear stresses. ${ }^{6}$ The interaction can be explained with the aid of the schematic sequence shown in Fig. 1. A vortex ring in free flight approaching a heated wall will interact ultimately with the thermal (and dynamic) boundary layer rather than the wall itself. During this interaction, the relevant points are the following: (i) diameter increases and vortex stretching is still observed as the ring approaches the wall; (ii) the boundary layer is compressed by the vorticity field during the collision; (iii) due to the ring rotation, adverse pressure gradients can produce boundary layer separation; (iv) the rolling-up of the boundary layer around the primary vortex takes place; and (v) finally, the vortex ring is convected upward by the natural convection flow and leaves the domain. The steady state of both the dynamic and thermal boundary layer is then restored.

\footnotetext{
a) Author to whom correspondence should be addressed. Electronic mail: rohernan@ing.uchile.cl. URL: http://www.leafnl.uchile.cl.
}

The heat transfer enhancement is observed mainly at the early stages of the impingement process in Fig. 1. High velocity gradients between the vortex ring and the flow near the wall are observed, and thus making a better mixing process by this interaction. For instance, Fabris et $a l^{2}{ }^{2}$ in a joint experimental-numerical investigation found that the peak vorticity grows about $50 \%$ by vortex stretching. Orlandi et $a .^{3}$ made numerical simulations, where peak vorticity of the boundary layer near the wall increases abruptly as the ring approaches the wall, eventually leading to boundary layer separation even at moderately high Reynolds numbers. This process has been accurately reported by Ref. 4, showing that secondary and tertiary vortices are originated by adverse pressure gradients between the primary vortex core and the wall. The formation and rolling-up of secondary vortices force a rebound of the primary one, as reported by Refs. 2, 3, 5 , and 7 . Such a variety of stages in the physical mechanism can be identified either by local vorticity or shear stress measurements, leading to a better understanding of the improvement of the heat transfer and mixing processes shown in Ref. 1. Since the collision produce local variations of vorticity and shear stresses near the wall, a detailed description of the vector velocity field during the collision must be accomplished. In the present work, we performed stereo particle image velocimetry (PIV) measurements of the ring velocity field leading to accurate vorticity and shear stresses computations during the ring collision with a heated wall. This paper is divided as follows: experimental setup is presented in Sec. II; results for the studied phenomena are presented in Sec. III. Concluding remarks are summarized in Sec. IV. 
1

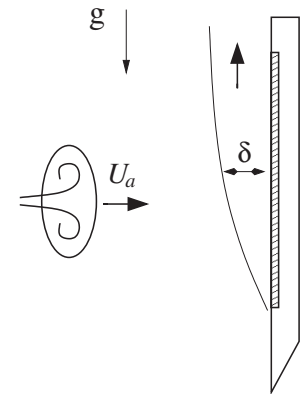

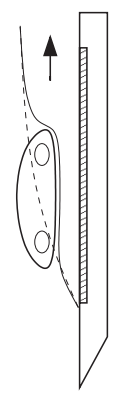

3

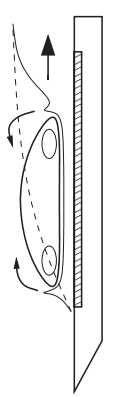

4

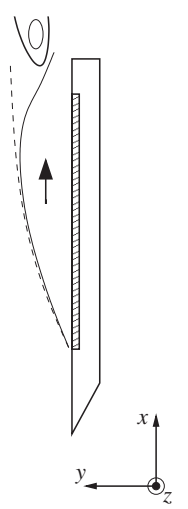

FIG. 1. Schematics of a vortex ring of self-induced velocity $U_{a}$ colliding with a thermal boundary layer of width $\delta$. (1) The vortex ring approaching the heated plate. (2) The boundary layer begins to be perturbed, (3) after that, boundary layer separation and entrainment of hot fluid into the vortex ring occurs. (4) Both the remanent ring and the perturbed boundary layer are convected away from the heater, and the natural convection regime is restored.

\section{EXPERIMENTAL SETUP}

The experiment is sketched in Fig. 2. A vortex ring of diameter $D$ and self-induced velocity $U_{a}$ is created with a vortex ring generator made with a flat Plexiglas piston driven by a loudspeaker. The piston displacement $(\sim 0.5 \mathrm{~mm})$ pushes an air-slug volume through an exit orifice of diameter $D_{0}=10 \mathrm{~mm}$. Different vortex rings properties can be obtained through simple modifications of the characteristic time of the signal $s(t)$ used to drive the loudspeaker (more details in Refs. 1 and 8). Each vortex ring will be characterized by its Reynolds number, $\operatorname{Re}=U_{a} D_{0} / \nu$ where $\nu$ is the kinematic air viscosity. In order to have a vertical heated wall, a flat square heater (size $H=5 \mathrm{~cm}$ ) was mounted flush over a larger and high-density fiberboard plate, as shown in Fig. 2. The heater, made of a thin Cermet film deposited on a Kyocera substrate, is maintained at a constant temperature $\left(T_{w}=50{ }^{\circ} \mathrm{C}\right)$ by electronic feedback. The vortex ring generator was placed facing the heater center separated by a con-
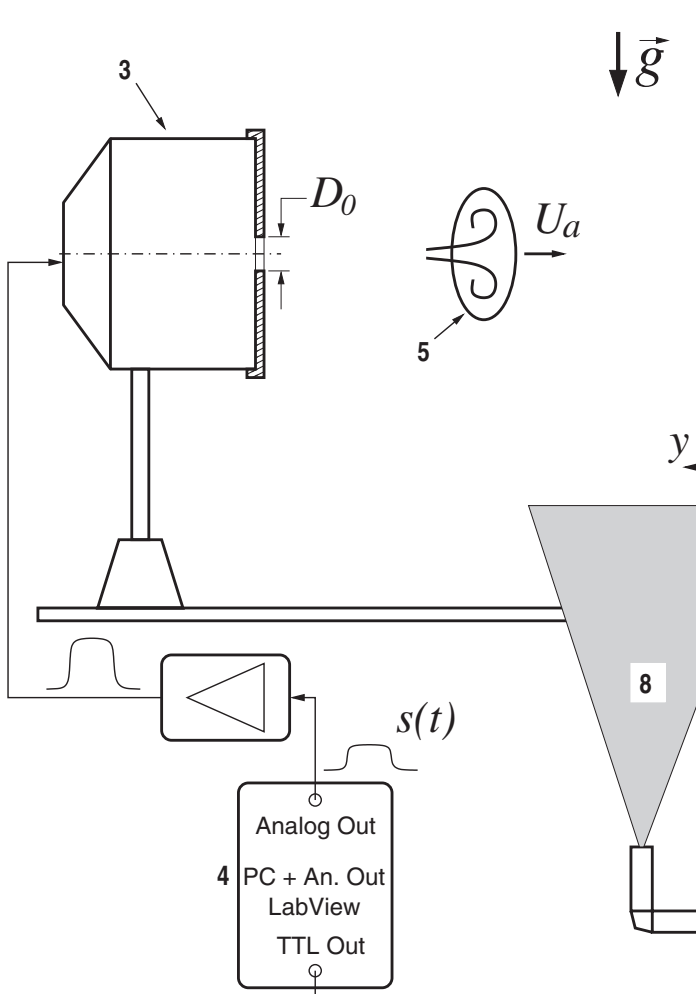

2

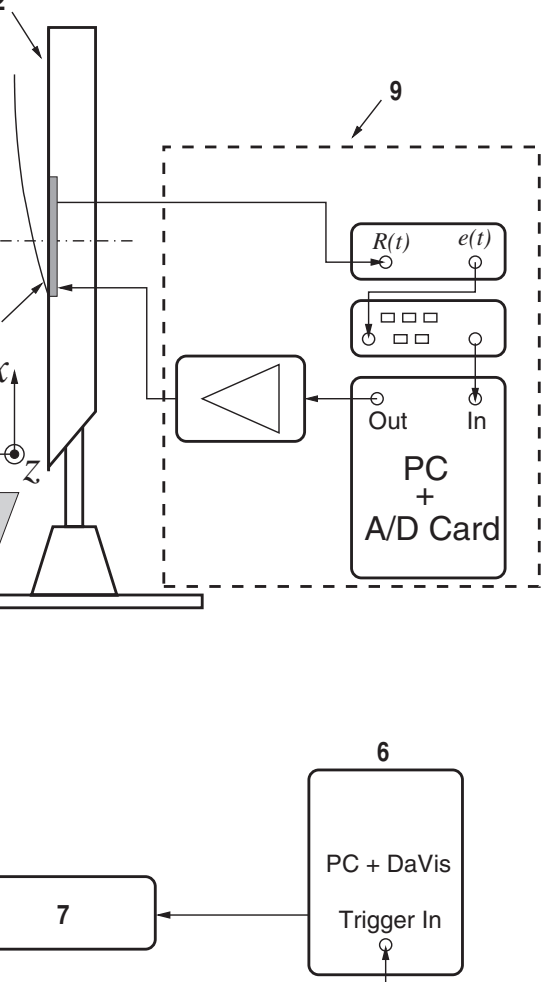

FIG. 2. Experimental setup. The heated plate (1) was mounted flush over a fiberboard vertical wall (2). The vortex ring generator (3) placed at a fixed distance $L=100 \mathrm{~mm}$ from the vertical wall was aligned with the center of the heater. A computer (4) with a NI PCI-6713 D/A card and a power amplifier were used to drive the ring generator. When a vortex ring (5) is generated, a TTL output triggers the PIV system (6), which drives a NewWave Solo PIV III pulsed laser, (7) thus generating a $4 \mathrm{~mm}$ laser sheet (8) for particle visualization. This laser sheet can be aligned with the $x y$ plane (at the ring's axis) or the $x z$ plane (at $6 \mathrm{~mm}$ from the vertical wall). The squared heated plated (50 mm width) was maintained at a constant temperature of $T_{w}=50{ }^{\circ} \mathrm{C}$ with a digital PI controller (9). 

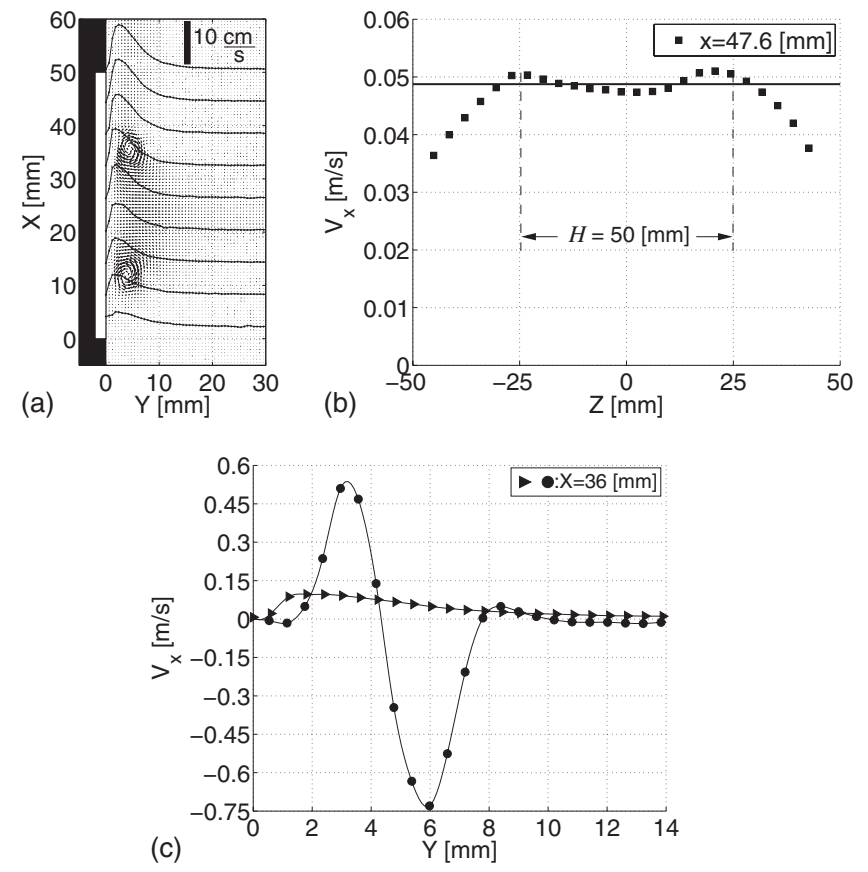

FIG. 3. (a) The velocity profile of the unperturbed free-convection flow originated by the heater, superimposed to the vector velocity field of the vortex ring impingement process, for $\mathrm{Re}=500$ with heater turned on. (b) Velocity profile of the boundary layer generated by the heater, along the $z$ coordinate, at $y=6 \mathrm{~mm}$ from the heated plate (uniformity of $\pm 5 \%$ along heater width $H$ ). (c) Comparison of vertical velocity profiles for both the unperturbed boundary layer $(\checkmark)$ and the vortex ring ( $)$ taken from (a) at $x=36 \mathrm{~mm}$.

stant distance of $L=100 \mathrm{~mm}$. Under this configuration, vortex rings travel in a free flight toward the wall, finally colliding with the heater. In order to avoid undesired air currents, both the vortex generator and the vertical wall were placed inside a larger closed glass box. The PIV measurements were done with a LaVision PIV system based on the following components: a NewWave Solo PIV III-15, $532 \mathrm{~nm}$ and $50 \mathrm{~mJ}$ pulsed laser, two Sensicam cooled imaging charge coupled device cameras, 12 bits, $1280 \times 1024$ resolution, and a PC with DAVIS software to record and process PIV images. A transistor-transistor logic (TTL) signal allows to synchronize the PIV with the vortex generator to capture the velocity field at different stages of the impingement process. The PIV seeding system produced seeding particles that can last $4 \mathrm{~h}$ with no appreciable stratification, ensuring a good particle homogeneity over the whole visualization area. Every PIV measurement was performed $1 \mathrm{~h}$ after the seeding process to minimize air currents resulting from.

In the simplified scheme of the PIV system in Fig. 2, the laser sheet is parallel to the $x y$ plane and aligned with the center of the heater. This configuration allows to measure the velocity field in a plane passing through the ring's symmetry axis. However, it is possible to rotate the laser sheet to make it parallel to the vertical wall in order to obtain a front view of the velocity field during the impingement process. In this configuration, the laser sheet and the vertical wall are $6 \mathrm{~mm}$ apart to avoid undesired light reflections from the wall.
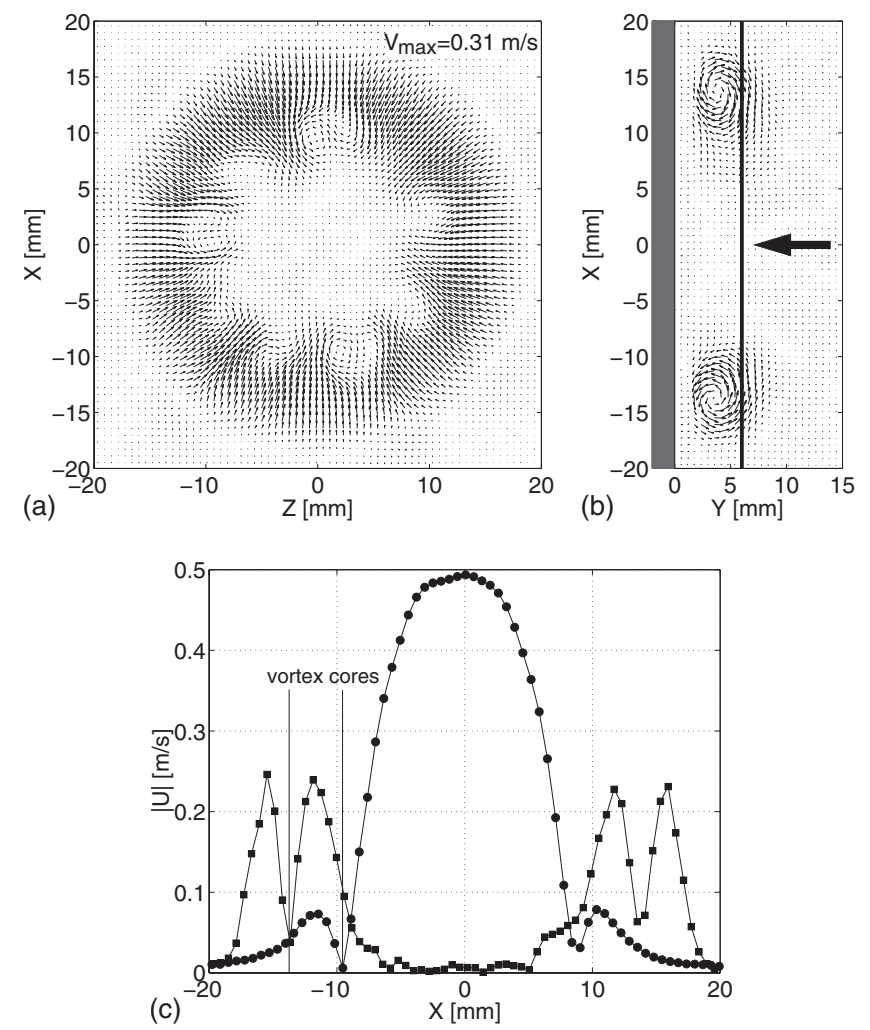

FIG. 4. Velocity field without heating at $R e=400$. (a) Velocity field in a plane parallel to the vertical wall where the laser sheet is $6 \mathrm{~mm}$ apart from the wall to avoid light reflections. (b) Velocity field in a plane perpendicular to the wall, along the vertical axis and at the center of the impingement region. The vertical line indicates the laser sheet position for figure (a). The gray-filled area is the vertical wall. (c) Velocity profiles of absolute velocity for $\operatorname{Re}=400$, when a ring is in free flight $(\mathbf{)})$ at $y=100 \mathrm{~mm}$ from the wall and during impact $(\square)$ at $y=6 \mathrm{~mm}$ from the wall. Vortex core positions are indicated by vertical lines

\section{RESULTS}

Prior to vortex ring/heated plate interaction, we measured the velocity field of the boundary layer generated by the vertical heater in a laminar natural convection regime at constant temperature $T_{w}=50{ }^{\circ} \mathrm{C}$. The Grashof number of the system based on the height $H$ of the heater is

$$
G=\frac{g H^{3}\left(T_{w}-T_{\infty}\right)}{\nu^{2} T_{\infty}} \sim 10^{5},
$$

where $g$ is the gravitational acceleration, $T_{\infty}$ and $\nu$ are the temperature and kinematic viscosity of the surrounding air, respectively. At this value of $G$, a stable, laminar boundary layer is obtained. ${ }^{9}$

In order to characterize the dynamic boundary layer for this regime, PIV measurements were performed along the $x y$ and $x z$ planes, as described in Sec. II. For every measurement plane, of the order of 100 different realizations were ensemble averaged to obtain a characteristic velocity field. For both cases, velocity fluctuations (in terms of the rms value) are less than 5\%, confirming that the boundary layer is laminar and stable.

In Fig. 3, we show velocity profiles ( $x y$ and $x z$ planes) for the unperturbed natural convection flow generated by the heater plate and the vector velocity field associated to the 
(a)
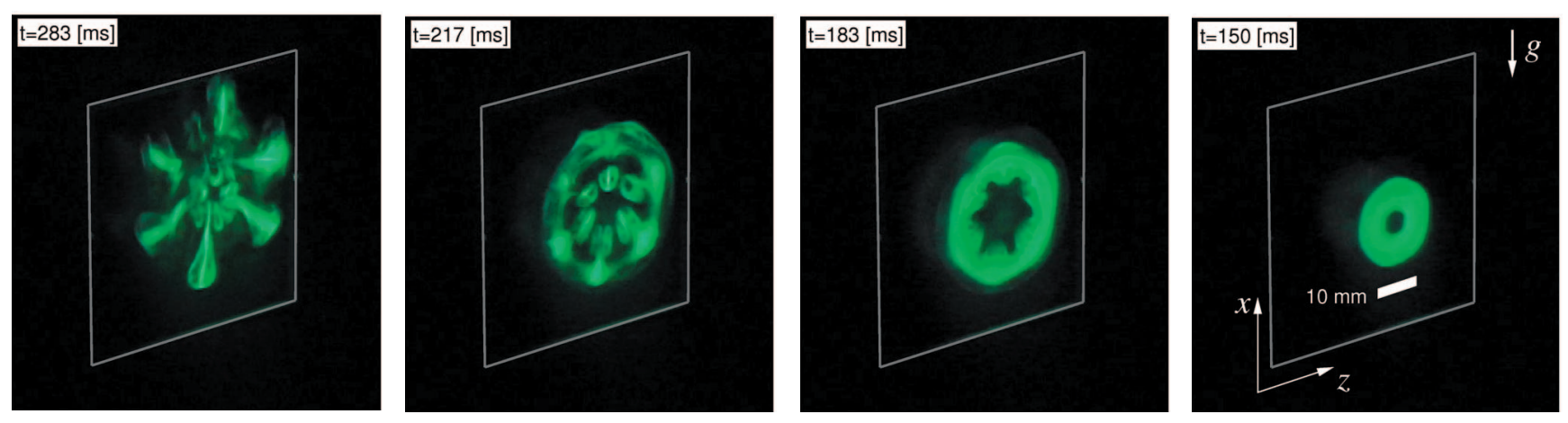

(b)
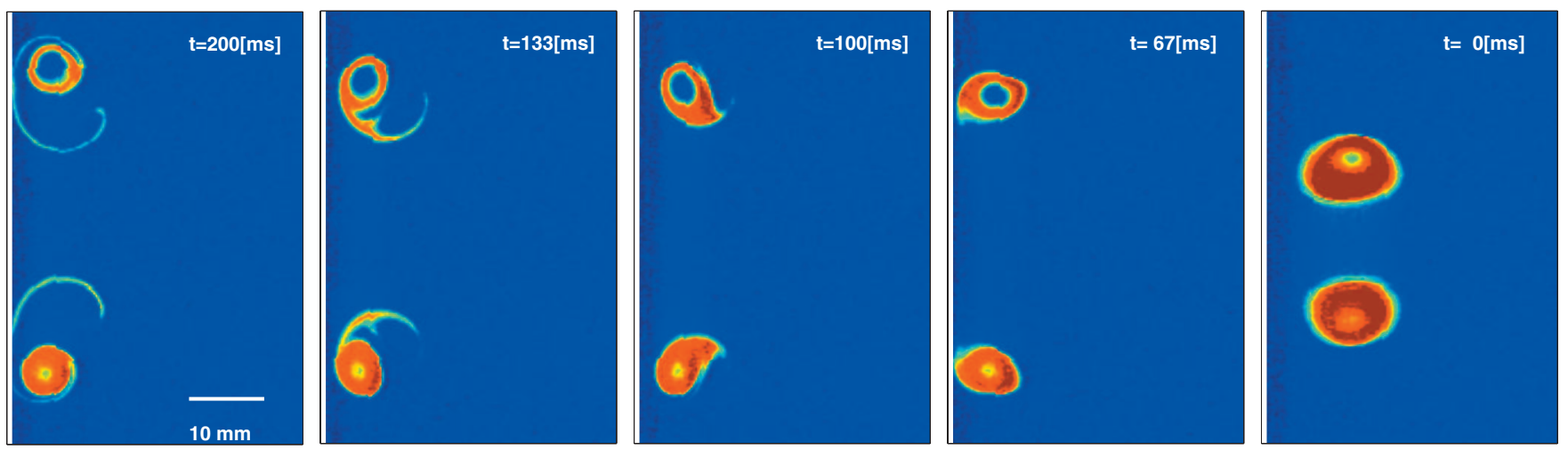

FIG. 5. (Color) Flow visualizations without heating at (a) $\mathrm{Re}=500$ and (b) $\mathrm{Re}=400$ using a 532 nm laser sheet. The vortex ring has been filled with smoke during the generation process. (a) The laser sheet is at $6 \mathrm{~mm}$ from the heated wall and time starts at the beginning of the generation process. Gray lines represent the heater position and dimensions. (b) Side view at midplane during collision where the ring stays glued to the wall during many turnover times producing an effective wall shear stress profile, peaked in the circumference of the ring.

vortex ring at $\mathrm{Re}=500$. We collected the upward progression of velocity profiles of a stable and unperturbed boundary layer measured with the PIV setup and then we measured the velocity field during ring impingement, superimposing the vector field with the velocity profiles of the boundary layer as in Fig. 3(a).

Figure 3(c) shows individual plots of velocity profiles taken from figure (a) corresponding to both the boundary layer and to the vortex ring. The idea is to compare typical spatial and velocity scales. The full width at half maximum (FWHM) of the velocity profile for the dynamic boundary layer is similar to the core size of the vortex ring in that plane. However very different peak velocities are observed during the impingement process, as shown in Fig. 3(c). The up-down asymmetry of the boundary layer makes the ring impingement also nonsymmetrical. The upper part of the boundary layer is thicker than the lower part, with higher velocities. The lower part of the ring arrives closer to the heated wall than the upper part. In Fig. 3(b), we show the extension and uniformity of a velocity profile along $z$ coordinate recorded at $y=6 \mathrm{~mm}$ from the heater. In front of the heater zone, $-H / 2<z<H / 2$, we have a flow uniformity of about $5 \%$. Outside the heater zone the flow velocity falls down rapidly due to the lack of buoyancy, as this portion of the wall is inactive. First of all, we make a set of PIV mea- surements of the velocity field during the impingement process without heating. The idea was to be sure of the capabilities of the PIV system in determining the spatial vortex structures originated during the collision. We measured the vortex velocity field at different time instants, for both $x y$ and $x z$ planes and for three ring Reynolds numbers Re $=U_{a} D_{0} / \nu$.

A typical vector velocity field for both planes is presented in Fig. 4 at $\mathrm{Re}=400$. The front view of the ring's velocity field in Fig. 4(a) shows important spatial variations in the azimuthal direction during the impact. However, inside the central ring area, velocity fluctuations become very small. The midplane side view of the ring's vector velocity field in Fig. 4(b) shows two zones of fluid rotation around the vortex ring core that allow to estimate the rapid rate of increase in the ring's diameter. The ring approaches the wall reducing its axial velocity, increasing both its diameter and peak velocity around the vortex core. This last progressive effect can be seen in Fig. 4(c), where we plot the ring's midplane velocity profile in free flight and close to the wall. The center velocity reduction between the free flight ring and the colliding one is significative, accompanied with the corresponding ring's diameter increase. We plotted absolute velocity to easily identify the position of the vortex core in the midplane, indicated by vertical lines, to measure ring diam- 


\section{Heater OFF}

(a)

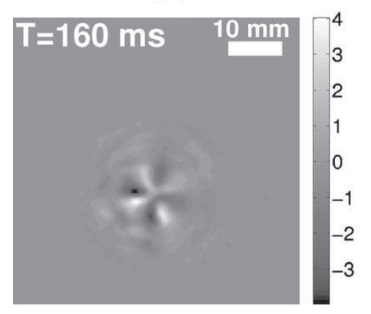

(c)

Heater ON

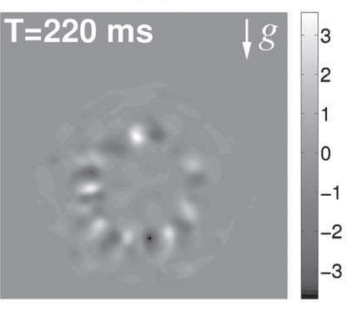

(d)

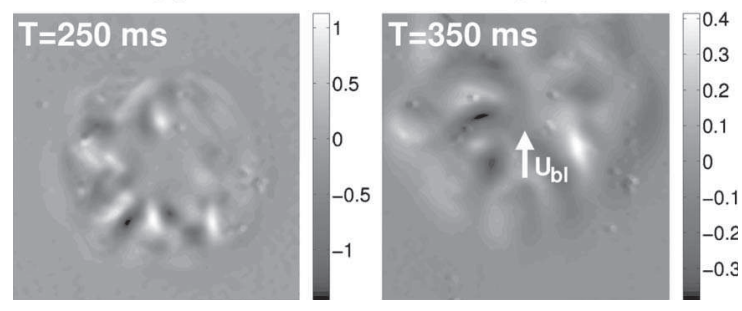

(f)

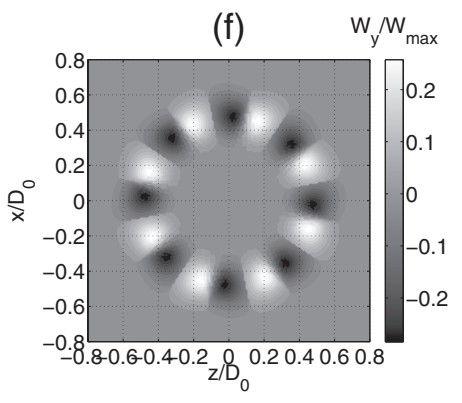

(e)

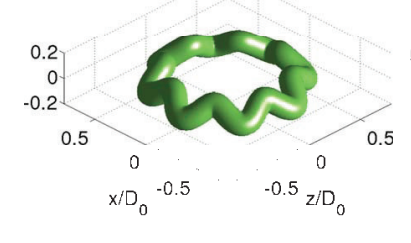

FIG. 6. (Color online) Flood contours of the vorticity field $\omega_{y}$ in the $x z$ plane [see, for instance, Fig. 4(a)] at different time instants of the impingement process, for $\mathrm{Re}=400$. (a) Heater off for $t=160 \mathrm{~ms}$. (b) Heater off for $t$ $=220 \mathrm{~ms}$. (c) Heater on for $t=250 \mathrm{~ms}$. (d) Heater on for $t=350 \mathrm{~ms}$. (e) Isosurface of vorticity values (50\% of maximum vorticity) for a constant vorticity distribution along the perturbed core axis. (f) Vorticity $\omega_{y}$ normalized by the maximum vorticity value, along the $x y$ in the middle plane of the ring.

eter. The net increase in peak velocity around the vortex core and the radical reduction of center velocity zone, suggest that it is the vortex core region responsible for important wall shear stresses. The velocity profile in free flight was also recorded with a hot wire anemometer showing no significative differences with the PIV velocity profile.

The spatial structures observed during the impingement process of initially stable vortex rings are the result of a ring instability identified as a particular flow arrangement of the velocity field under vortical structures ordered in the azimuthal coordinate. It is important to say that if we take off the wall, the vortex ring velocity field does not display any distortion away from a typical ring velocity field.

We can verify this result by means of the flow visualizations shown in Fig. 5. We filled with smoke a vortex ring $(\mathrm{Re}=500)$ during the generation process and then we illuminated with a $532 \mathrm{~nm}$ laser sheet $(2 \mathrm{~mm}$ width) placed parallel to the $x z$ axis, at a distance of $6 \mathrm{~mm}$ from the vertical wall. Top images were recorded with a JVC GR-D32 interlaced digital camera at a framerate of $30 \mathrm{~Hz}$, where only the upper fields were used to avoid interlacing issues. Bottom images were recorded with a Cohu 6600-3000, 10 bit, and $60 \mathrm{fps}$ digital camera.

Flow visualizations in Fig. 5 confirm that the initially unperturbed vortex ring becomes unstable during the impingement process. As indicated by the elapsed-time inset, at $t=183 \mathrm{~ms}$, when the ring starts to hit the wall, a six-arm azimuthal starlike periodic pattern begins to develop, probably originated by Widnall's instability. ${ }^{10,11}$ At $t=217 \mathrm{~ms}$, the starlike structure is well developed and evolves into vortical structures around the center of the impact. At $t$ $=283 \mathrm{~ms}$ the smoke used to trace the ring instability, is mainly concentrated along the arms of the starlike pattern, making visible the rapid increase in the arms. This is an important issue to keep in mind when we consider the mixing capabilities of a ring; the tracer is being concentrated into a periodical azimuthal pattern instead of a typical circle. At this point the natural question is to ask weather or not these arms are the only vortical structures that remained from the impinging circular vortex ring?

To answer that question we need to compute the vorticity field from the PIV velocity records at different time instants. Two vorticity components $\left(\omega_{y}, \omega_{z}\right)$ were computed from the velocity field by means of a standard central difference scheme on each vector velocity matrix, where the grid spacement used in the PIV measurements was $\Delta x=\Delta y$ $=\Delta z=0.63 \mathrm{~mm}$.

These results, summarized in Fig. 6, show the dimensionless (normalized by $U_{a} D_{0}^{-1}$ ) vorticity magnitude $\omega_{y}$ at different time instants of the impingement process, at Re $=400$ with the heater-off, in Figs. 6(a) and 6(b), and with the heater-on, in Figs. 6(c) and 6(d). It is observed the formation of vortex structures with an important component of $\omega_{y}$ vorticity, i.e., vorticity in the direction normal to the wall. If the heater is off, the initial flood contours of $\omega_{y}$ vorticity arrange into an azimuthal starlike vortex pattern, containing eight vortex zones; four with positive vorticity which alternate with four zones of negative vorticity. As the time increases, these vortex structures grow in number, increasing the overall size of the circular spot. The process is accompanied by an important reduction of peak vorticity in such a spatial plane, presumably by the effect of viscous dissipation, as shown in Fig. 6(e).

A similar effect is observed with the heater-on, but the alternated vortex zones are principally formed in the lower portion of the impact zone, where the local vorticity of the boundary layer has the same sign than the lower portion of the vortex ring. This up-down asymmetry during the impingement process is much more evident when the vortical structures are finally convected upward by the thermal boundary layer, as part of the last stages of the ring/wall interaction in Figs. 6(c) and 6(d).

To understand qualitatively the vorticity plots of Fig. 6 and the creation of vorticity in the direction normal to the wall, $\omega_{y}$, we made use of the geometrical model developed by Dazin. ${ }^{12}$ The model gives an expression for the core axis of an unstable ring in order to mimic the results of Widnall ${ }^{10}$ on the azimuthal instability of vortex rings. The parameter identifying a vortex ring is the dimensionless velocity $\widetilde{U}$ 


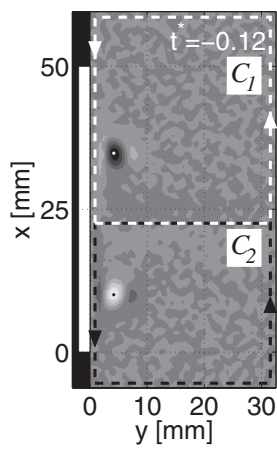

(a)

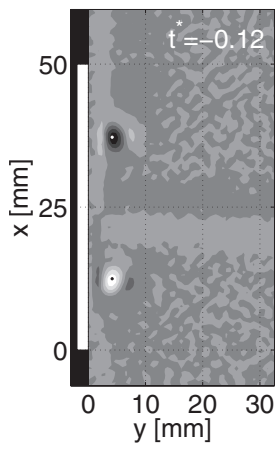

(d)

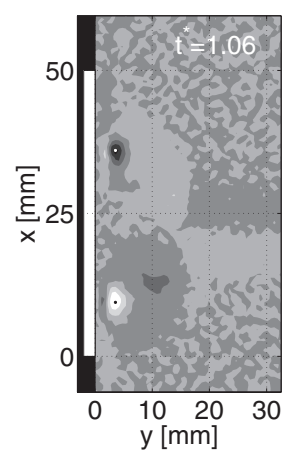

(b)

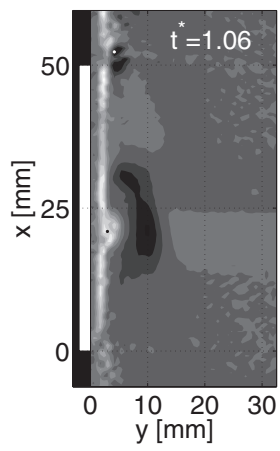

(e)
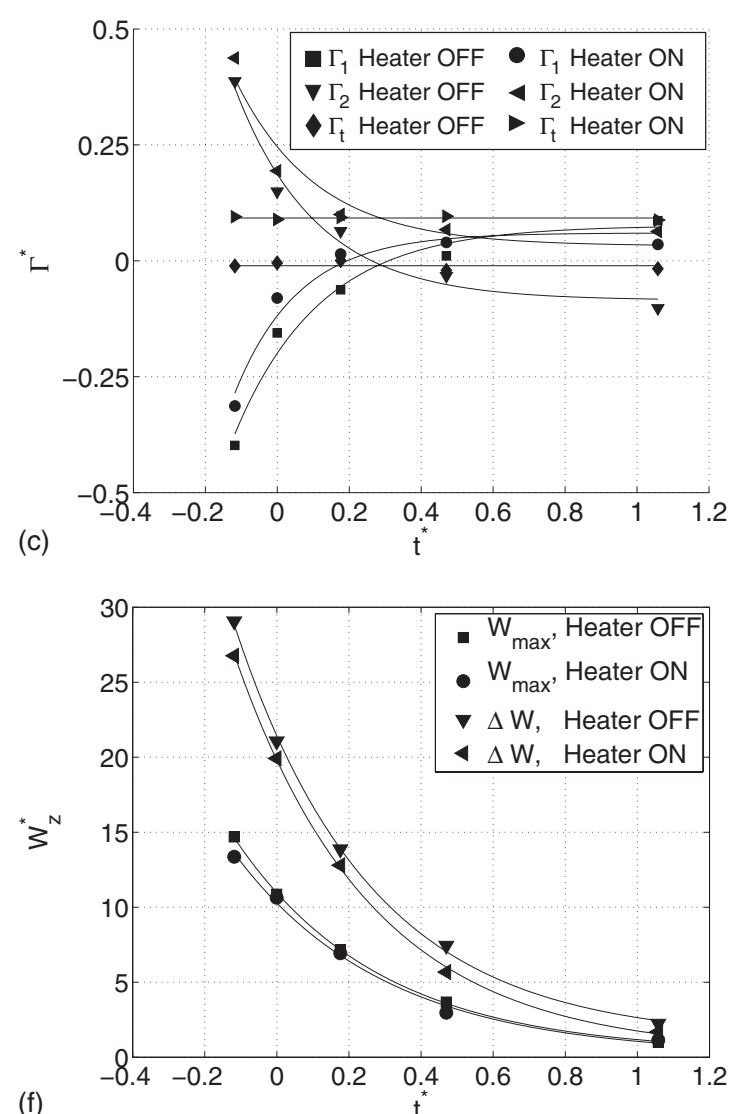

FIG. 7. Flood contours of vorticity magnitude $\omega_{z}(x, y)$ when the heater is off $[(a)$ and (b)] and when the heater is on [(d) and (e)], during the impingement process, for $\mathrm{Re}=400$. Black/white dots indicate the location of maximum/minimum vorticity inside both upper and lower vortex ring cores, respectively. The vertical wall is shown in black color (left border) and the heater is represented by a white rectangle. (c) Dimensionless circulation $\Gamma_{1}$, $\Gamma_{2}$, and $\Gamma_{t}$ computed in the paths shown in (a). (f) The evolution of the dimensionless maximum vorticity with time.

$=4 \pi R U_{a} / \Gamma$ from what the number of instability waves $n$ can be computed. For a constant vorticity tube, a torus of radius $R$ and core radius $a$,

$$
\tilde{U}=\ln (8 R / a)-1 / 4 \text {. }
$$

We have evaluated $\widetilde{U}$ from PIV data at $\operatorname{Re}=400$, giving $\widetilde{U}$ $\sim 3$ where the number of unstable waves is $n \sim 8$. The geometrical model with $n=8$ produced the distorted vorticity torus of Fig. 6(e). When we display $\omega_{y}$ component at $y=0$ cutting plane, just in the middle of the ring on Fig. 6(f), the observed pattern is very similar to the experimental one during the head-on collision. It is a sequence of 16 alternating vortex structures along the azimuthal direction of the ring where the change in vorticity sign is given by the upper and lower portions of a single wavelength on the perturbed torus.

However, vorticity will not last forever because the viscous dissipation with the wall is very strong. In Fig. 7, we compare the time evolution of $\omega_{z}$ vorticity and circulation $\Gamma$, for $\operatorname{Re}=400$, when the heater is on and off. The black and white dots in the flood contours of Figs. 7(a), 7(b), 7(d), and 7 (e) represent the midplane vortex ring core approaching the wall. In both situations the vortex core remains at a finite $x$-distance from the wall, independent of the ring's Reynolds number. There is, however, a slight difference between both situations: In the heater-on case, the ring moves upward by the action of the natural convection boundary layer, and the black and white dots representing the vortex core are not equally apart from the heated wall. The lower black dot is closer to the wall than the upper white dot, presumably an effect of the differences in boundary layer width from top to bottom.

This rapid decay of vorticity during the impingement process is also observed in the circulation from the upper and lower vortex cores. The PIV data field allows to compute the circulation $\Gamma$ with the following expressions:

$$
\begin{aligned}
\Gamma_{1} & =\int_{C_{1}} \mathbf{u} \cdot d \mathbf{l}=\int_{A_{1}} \boldsymbol{\omega} \cdot d \mathbf{A}=\int_{A_{1}} \omega_{z} d A \\
& \sim \sum_{i \in A_{1}} \sum_{j \in A_{1}} \omega_{z}(i, j) \Delta x \Delta y, \\
\Gamma_{t} & \sim \sum_{i \in A_{t}} \sum_{j \in A_{t}} \omega_{z}(i, j) \Delta x \Delta y, \quad A_{t}=A_{1} \cup A_{2} .
\end{aligned}
$$

In Fig. 7 , the circulation $\Gamma_{1}, \Gamma_{2}$ where computed with the paths $C_{1}, C_{2}$ enclosing the upper and lower vortex core, respectively, and both passing through the symmetry axis of the vortex ring. The total circulation $\Gamma_{t}$ was computed by considering the whole area $A_{t}$ enclosed by $C_{1}$ and $C_{2}$. Figure 7 (c) shows the evolution of dimensionless circulation $\Gamma_{1}, \Gamma_{2}$, and $\Gamma_{t}$ with time $(\operatorname{Re}=400)$, where the circulation and vorticity scaling are $U_{a} D_{0}$ and $U_{a} D_{0}^{-1}$, respectively, and the di- 

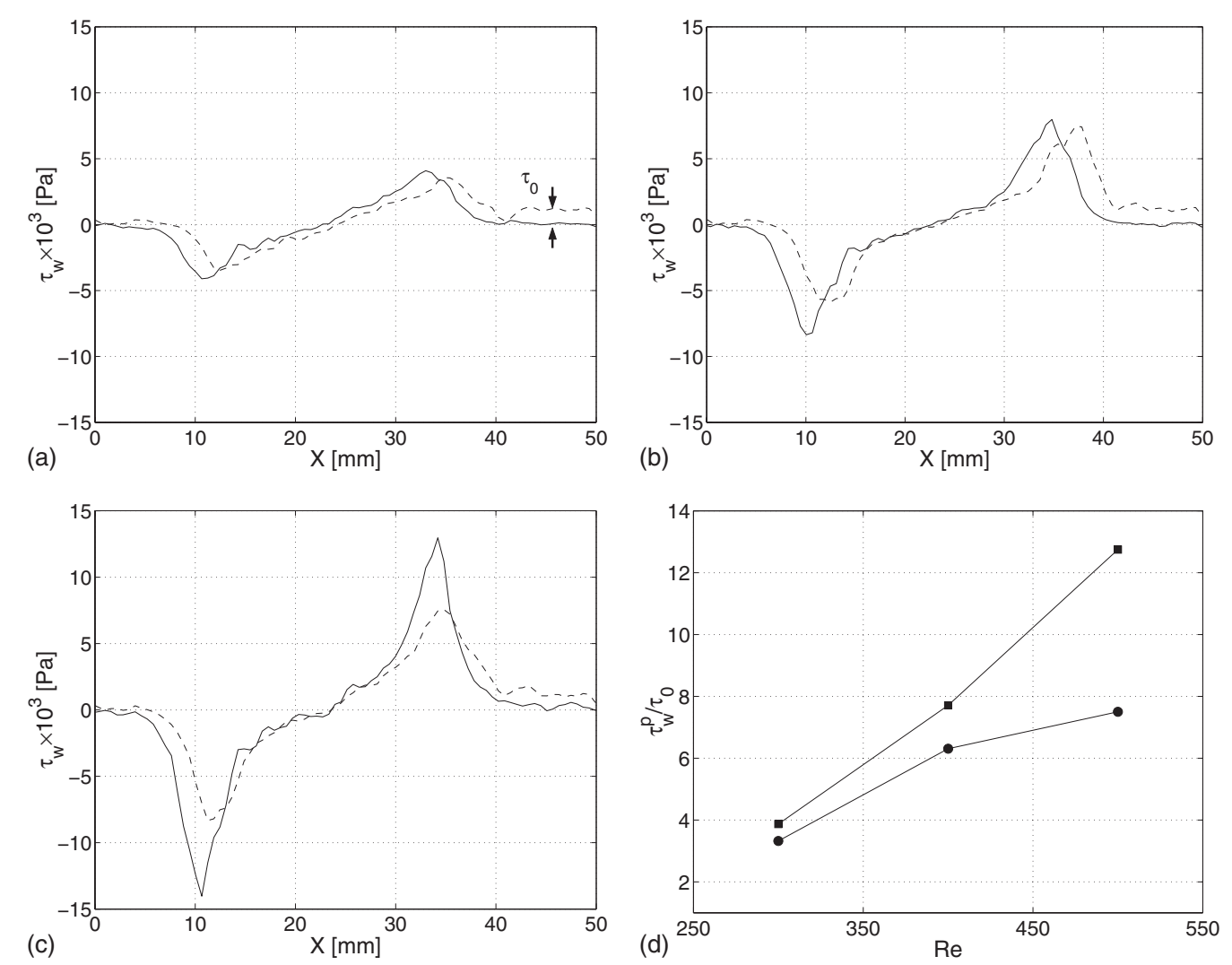

FIG. 8. Wall shear stress profile, $\tau_{w}(x)$, at the same time instant during collision, computed when the vortex ring core is 5 mm from the wall (heater-off continuous line). (a) $\operatorname{Re}=300$, (b) $\operatorname{Re}=400$, and (c) $\operatorname{Re}=500$. (d) Evolution of peak wall shear $\tau_{w}^{p}$ as a function of $\operatorname{Re}$ when heater is turned on $(\mathbf{a})$ and off ( (uncertainty of $4 \%$ ).

mensionless time axis was defined as $t^{*}=U_{a} t L^{-1}$. If the heater is off, there is no boundary layer and therefore no constant vorticity associated. The total circulation $\Gamma_{t}$ remains constant around zero. Upper and lower circulations, $\Gamma_{1}$ and $\Gamma_{2}$, display a similar evolution and similar magnitude. However, when the heater is on, the dynamic boundary layer adds a contribution to the total circulation $\Gamma_{t}$, a kind of vorticity offset which remains constant during the collision. This effect is also encountered when we determine the wall shear stresses produced by the ring impact with the aid of our PIV velocity data.

To compute these velocity gradients and therefore to determine local shear stresses at different stages of the collision we must have velocity data records taken very close to the wall. The ability to make such computations with and without heating, help us to distinguish the additional shear produced by the thermal boundary layer itself in the heater-on case. Shear stresses were computed along a vertical line passing through the center of the heater and defined as follows:

$$
\tau_{w}=\mu\left(\frac{\partial V_{x}(x, y, z=0)}{\partial y}\right)_{y=0},
$$

where $\mu$ is the dynamic air viscosity. ${ }^{13}$ The spatial velocity derivative was computed using a fourth-order polynomial fit for the velocity data points near the wall (six points total), for each $x$ location.

Figures 8(a)-8(c) show the value of $\tau_{w}$ along the $x$ co- ordinate for the impingement of stable vortex rings at three different Reynolds numbers. Shear stress profiles show two local peaks occurring near the point of impact of the upper and lower vortex ring cores $(x \sim 10$ and $x \sim 35 \mathrm{~mm}$, respectively) with a linear behavior in between, passing through zero at the symmetry axis of the impact. Outside the ring's impact area, there is a residual shear stress $\tau_{0}$ originated by the local vorticity of the boundary layer as already discussed in Fig. 7(c). However, $\tau_{0}$ is not observed inside the ring's impact area although it represents $25 \%, 14 \%$, and $8 \%$ of peak wall shear without heating at $\operatorname{Re}=300,400,500$, respectively. The origin of both local peaks is attributed to the steeper velocity gradients created by the vortex ring core, and the linear central region a consequence of the rapid expansion of the ring's diameter.

These results reinforce the idea that during the collision, the ring growth is accompanied by an increasing surface of impact on the plate, where the circular peaked wall shear stress profile performs a progressive swept of the boundary layer, thus increasing heat transfer area. There is an evident upward shift of the shear stress profile in the heater-on condition which is originated by the overall ring vertical motion produced by the convection flow. The distance between shear stress peaks, in both cases remains unchanged, with no noticeable differences in amplitude except in the case of Re $=500$. At this Reynolds number, the relative position between wall shear stress peaks is similar in both cases, meaning that the upward ring motion before impact is negligible. 
As $\tau_{0}$ is the wall shear associated to the boundary layer, a global measure of the colliding ring effect is to compare the peak values of wall shear stress $\tau_{w}^{p}(y \sim 0)$ with $\tau_{0}$ in both situations. Without boundary layer, the wall shear stress peak grows almost linearly with the ring Reynolds number Re. This behavior is however progressively damped in the presence of the boundary layer. The boundary layer clearly attenuates the collision process between the ring and the wall, but in that process the boundary layer cannot damp the impact completely without being destroyed. Such an attenuation is accompanied by a rapid decay of transverse vorticity $\omega_{z}$ but at the same time with the creation of normal vorticity $\omega_{y}$.

Note that the width of each peak correlates well with the size of the ring's core, as it can be corroborated with the core size of the colliding ring velocity profile in Fig. 4.

\section{CONCLUDING REMARKS}

We investigated the head-on collision of vortex rings on a vertical heated wall with traditional PIV velocity measurements, where our results indicate the following:

(i) The impingement process is divided into two main parts: An initial ring-driven stage where the ring approaches the wall, growing in diameter with an abrupt vorticity decay for either a heater-on or a heater-off case. A second and final boundary layer-driven stage, where the vortex ring, which has lost part of energy during the collision, is convected upward by the action of the convective motion of the boundary layer.

(ii) The impingement process at $\mathrm{Re}=400$ reveals the presence of Widnall's azimuthal instability, with the development of vortex structures in the azimuthal direction of the ring, as suggested by a geometrical model of the instability. These structures have vorticity normal to the wall, $\omega_{y}$, and they are observed in both situations, with heater on and off.

(iii) Wall shear stresses were computed along the vertical axis of the heater for different Reynolds numbers. At low Reynolds numbers both heater-on and heater-off cases exhibit similar spatial behavior. Wall shear stresses profiles show two local peaks occurring near the point of impact of the upper and lower vortex ring cores and passing through zero at the symmetry axis of the impact. There is an evident upward shift of the shear stress profile in the heater-on condition which is originated by the overall ring vertical motion produced by the convection flow.

(iv) Our results reinforce the idea that during the collision, the ring growth is accompanied by an increasing surface of impact on the plate, where the circular peaked wall shear stress profile performs a progressive radial swept of the boundary layer with the corresponding increase in the heat transfer area.

\section{ACKNOWLEDGMENTS}

This work was supported by Fondecyt Grant No. 1085020. G.A. acknowledges Mecesup Grant No. UCH0310 and wishes to thank Ecole Centrale de Lyon for kind hospitality and for the lending of the PIV system.

${ }^{1}$ G. Arévalo, R. H. Hernández, C. Nicot, and F. Plaza, "Vortex ring head-on collision with a heated vertical plate," Phys. Fluids 19, 083603 (2007).

${ }^{2}$ D. Fabris and D. Liepmann, "Quantitative experimental and numerical investigation of a vortex ring impinging on a wall," Phys. Fluids 8, 2640 (1996).

${ }^{3}$ P. Orlandi and R. Verzicco, "Vortex rings impinging on walls: Axisymmetric and three-dimensional simulations," J. Fluid Mech. 256, 615 (1993).

${ }^{4}$ A. Naguib and M. Koochesfahani, "On wall-pressure sources associated with the unsteady separation in a vortex-ring/wall interaction," Phys. Fluids 16, 2613 (2004).

${ }^{5}$ C. Chu, C. Wang, and C. Chang, "A vortex ring impinging on a solid surface-Vortex structure and surface force," Phys. Fluids 7, 1391 (1995).

${ }^{6}$ P. Abry, S. Fauve, P. Flandrin, and C. Laroche, "Analysis of pressure fluctuations in swirling turbulent flows," J. Phys. II 4, 725 (1994).

${ }^{7}$ P. Orlandi, "Vortex dipole rebound from a wall," Phys. Fluids A 2, 1429 (1990).

${ }^{8}$ R. H. Hernández, B. Cibert, and C. Béchet, "Experiments with vortex rings in air," Europhys. Lett. 75, 743 (2006).

${ }^{9}$ H. Schlichting, Boundary Layer Theory (McGraw-Hill, New York, 1968).

${ }^{10}$ S. E. Widnall and C.-Y. Tsai, "The instability of the thin vortex ring of constant vorticity," Philos. Trans. R. Soc. London, Ser. A 287, 273 (1977).

${ }^{11}$ T. Maxworthy, "The structure and stability of vortex rings," J. Fluid Mech. 51, 15 (1972).

${ }^{12}$ A. Dazin, P. Dupont, and M. Stanislas, "Experimental characterization of the instability of the vortex ring. Part I: Linear phase," Exp. Fluids 40, 383 (2006).

${ }^{13}$ D. Lide, CRC Handbook (CRC, Cleveland, 2002). 